\title{
Exploring semiconductor substrates for Silicene epitaxy
}

\author{
Amrita Bhattacharya $^{\dagger}$, Saswata Bhattacharya ${ }^{\dagger}$, Gour P. Das* \\ Dept. of Materials Science, Indian Association for the Cultivation of Science, Kolkata-32, India
}

(Dated: March 10, 2021)

\begin{abstract}
We have carried out first-principles based DFT calculation on electronic properties of silicene monolayer on various (111) semi-conducting surfaces. We find that the relative stability and other properties of the silicene overlayer depends sensitively on whether the interacting top layer of the substrate is metal or non-metal terminated. The nature of silicene-monolayer on the metal terminated surface can be metallic or even magnetic, depending upon the choice of the substrate. The silicene overlayer undergoes n-type doping on metal terminated surface while it undergoes p-type doping on non metal terminated surfaces of the semiconductor substrates.
\end{abstract}

Keywords: Silicene, Planar Nanostructures, Density Functional Theory, Electronic Structure

From first principles calculations, the 2D mono-layers of Silicon, known as silicene, has also been predicted to be stable [1-3] with graphene like semi-metallic characteristics viz. a linear dispersion relation at the $\mathrm{K}$ point $[4,6]$. The epitaxial growth of $\mathrm{Ag}$ thin film on $\mathrm{Si}(111)$ substrate has been explored by experimentalists in the past. The limited intermixing between $\mathrm{Ag}$ thin film and $\mathrm{Si}(111)$ substrate, make the choice of substrate ideal, for the growth of $\mathrm{Ag}$ thin films, so, it was natural to conjecture a reverse case. Infact, the experimental evidence of formation of silicene on $\mathrm{Ag}(001), \mathrm{Ag}(110), \mathrm{Ag}(111)$ substrates has been reported by various groups [7-12]. The formation of silicene on $\mathrm{Ag}(110)$ substrate has also been studied using first principles methods by A. Kara et al [13, 14]. More recently, evidence of epitaxial Silicene on non silver $\mathrm{ZrB}_{2}-0001$ substrate [15] and $\operatorname{Ir}(111)$ substrate [16] has also been reported. Moreover, silicene monolayer on $\mathrm{Ag}(111)$ was found to undergo a phase transition to two types of mirror-symmetric boundary-separated rhombic phases at temperatures below $40 \mathrm{~K}$ by scanning tunneling microscopy [17. However, the possibility of existence of other suitable substrates for formation of silicene monolayer can not be ruled out and has not been exhaustively explored till date.

In this communication, we report from our first principles based calculations, the bonding, stability and electronic structure of silicene monolayer, when epitaxially grown on various Group II-VI and Group III-V semiconductor substrates viz. $\operatorname{AlAs}(111), \operatorname{AlP}(111), \operatorname{GaAs}(111)$, $\mathrm{GaP}(111), \mathrm{ZnS}(111)$ and $\mathrm{ZnSe}(111)$. The nature of silicene monolayer on the metal terminated surface of these substrates can be metallic or even magnetic, depending upon the choice of the substrate. The silicene overlayer undergoes n-type doping on metal terminated surface while it undergoes p-type doping on non metal terminated surfaces of the semiconductor substrates.

Our calculations have been carried out using firstprinciples density functional theory (DFT) [18, 19] based total energy calculations. We have used VASP [20] code with projected augmented wave (PAW) potential[21] and Perdew-Burke-Ernzerhof (PBE) exchange correla-

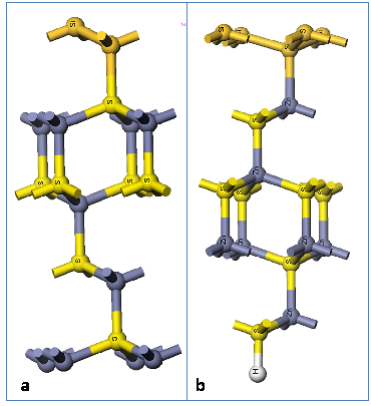

FIG. 1. Lateral view of one unit cell of epitaxial silicene on semiconductor substrate with $\mathrm{Si}$ atom attached to (a) S (nonmetal terminated) (b) Zn (metal terminated) surface of $\mathrm{ZnS}$ (111). The blue and yellow balls corresponds to the $\mathrm{Zn}$ and $\mathrm{S}$ atoms of the substrate, while the top overlayer is composed of silicene atoms.

tion functional[22] within generalized gradient approximation (GGA). An energy cut off of $600 \mathrm{eV}$ has been used. The $\underline{\mathrm{k}}$-mesh was generated by Monkhorst-Pack method and the results were tested for convergence with respect to mesh size $[8 \times 8 \times 1]$. However, for the generation of the electronic density of states (DOS) and band structure plots, higher values of $\underline{k}$-points $[16 \times 16 \times 1]$ were used. In all our calculations, self-consistency has been achieved with a $0.0001 \mathrm{eV}$ convergence in total energy. For optimizing the ground state geometry, forces on the atoms were converged to less than $0.001 \mathrm{eV} / \AA$ via conjugate gradient minimization 23 .

The silicene unit cell has a buckled hexagonal planar geometry, with a lattice parameter of $3.84 \AA$ and a homogeneous Si-Si bond distance of $2.29 \AA$ throughout the sheet. The semiconductor substrates viz. $\operatorname{AlAs}(111), \operatorname{AlP}(111)$, GaAs(111), GaP(111), ZnS(111) and ZnSe(111), that we have considered for epitaxial growth of silicene monolayer, have resonably good lattice matching with that of silicene, as given in TABLE-I. In this work, we have only considered the direct lattice matched non polar(111) substrates. However, other substrate orientations may also be relevant and will be addressed in our future 
TABLE I. Binding energy (in eV/atom) of Silicene monolayer to different semiconductor substrates having lattice constants close to that of silicene $(3.84 \AA)$.

\begin{tabular}{llll}
\hline Surface & $\begin{array}{l}\text { Lat. } \\
\text { const. }(\AA)\end{array}$ & $\begin{array}{l}\text { BE } \\
\text { Si@MT }\end{array}$ & $\begin{array}{l}\text { BE } \\
\text { Si@NMT }\end{array}$ \\
\hline $\operatorname{AlAs}(111)$ & 4.0038 & 0.630 & 0.370 \\
$\operatorname{AlP}(111)$ & 3.8544 & 0.685 & 0.910 \\
$\operatorname{GaAs}(111)$ & 3.9974 & 0.475 & 0.880 \\
$\operatorname{ZnS}(111)$ & 3.8249 & 0.570 & 0.675 \\
$\operatorname{GaP}(111)$ & 3.8541 & 0.605 & 0.525 \\
$\operatorname{ZnSe}(111)$ & 4.0076 & 0.440 & 0.540 \\
\hline
\end{tabular}

study.

Therefore, the unit cell of the composite system consists of the silicene monolayer (having two Si atoms) placed on top of six layers of the substrate (having 12 atoms), with a vacuum slab of $20 \AA$ above it. It is to be noted here that in these substrates, the contact layer of the substrate that interacts with the silicene overlayer could either be non metal terminated (NMT) or metal terminated (MT), e.g. in $\mathrm{ZnS}(111)$, the interacting layer could be either $\mathrm{S}$ terminated or Zn terminated (FIG 1). If the contact layer is NMT, then the MT bottom layer in the unit cell does not need H-passivation (FIG 1 1 ). However, if the contact is MT, then the lowest layer of the six layer substrate in the unit cell is NMT that needs to be passivated by hydrogen (FIG.1b), in order to avoid the effect of dangling bonds. The nomenclature used for designating an unit of NMT substrate is NM6M6, while the MT substrate is denoted as M6NM6H. For calculating the binding energy (BE) and magnetic interaction of the silicene monolayer with the semiconductor substrates we have used a $4 \times 4$ supercell. The BE of silicene monolayer to the MT and NMT surfaces of these substrates are summarized in TABLE-I. For MT surface the BE values lie in the range of $0.56 \pm$ $0.12 \mathrm{eV} /$ atom (TABLE-I) which is comparable to that of silicene monolayer on $\mathrm{Ag}(111)$ substrate, for which our estimated value $(0.52 \mathrm{eV} /$ atom $)$ is in good agreement with first principle result of P. Vogt et.al [17]. Thus, on comparing the binding energies of silicene to semiconductor MT/ NMT substrates with $\operatorname{Ag}(111)$, we find that they are close or comparable for metal terminated surfaces of $\mathrm{GaAs}(111), \mathrm{GaP}(111), \mathrm{ZnS}(111), \mathrm{ZnSe}(111)$ substrates and non metal terminated surfaces of $\operatorname{AlAs}(111)$, $\mathrm{GaP}(111)$ and $\mathrm{ZnSe}(111)$ substrates (TABLE-I).

Our calculations show that when the bulk substrate (both MT and NMT) is cleaved to certain number of layers, the top layer contains uncompensated dangling bonds and therefore, give rise to magnetic moment in the substrate (TABLE-II). We have estimated the magnetic moment of the substrate, before and after introduction of silicene monolayer which are given in TABLE-II. We would first discuss the behaviour of silicene with the NMT semiconductor substrates. When silicene mono- layer is introduced to the NMT surface of these substrates, the magnetic moment of the composite system gets enhanced. For the sake of simplicity, this magnetism can be thought upon as the effect of uncompensated dangling bonds at the surface of the substrate. However, the nature of charge transfer between the silicene layer and substrate may play a vital role in enhancement of magnetism of the composite system in all NMT cases. We have performed Bader charge analysis of NMT substrates before and after inclusion of silicene layer. In each case, the charge gets transferred from the silicene sheet to the contact atoms (viz. As, P, S, Se, Ge) of the NMT substrates (see TABLE-III), because of the later having higher electronegavity as compared to Si. Therefore, in all NMT cases the silicene overlayer undergoes p-type doping due to its interaction with the substrate.

In order to understand the interaction of silicene with these substrates and to help their characterization in the laboratory it is necessary to study the magnetic behavior of silicene with substrate. Therefore, we have plotted the layer projected DOS and the fattening of silicon bands near the Fermi level in order to study the interaction of the sheet with these substrates. Unlike the NMT cases, the behaviour of silicene monolayer on the MT surface can be divided into three categories. The introduction of silicene monolayer on MT surface, may quench/ enhance the magnetism or may not have any effect at all, on the magnetic moment of the composite (silicene + substrate) system as shown in TABLE-II. In case of $\operatorname{AlAs}(111)$, $\operatorname{AlP}(111)$ and GaAs(111) [Case-I], the surface magnetism of the MT surface of the substrate is quenched after introduction of silicene monolayer and as a result of this, the magnetic moment of the composite system is found to vanish. Whereas, in $\mathrm{GaP}(111)$ and $\mathrm{ZnSe}(111)$ [Case-II], the introduction of silicene monolayer enhances the magnetic moment of the composite system. However, only in case of $\mathrm{ZnS}(111)$ substrate [Case-III], the magnetic moment of the MT surface remains 'zero' before as well as after inclusion of the silicene monolayer.

The behaviour of silicene on MT surface of semiconductor substrates can also be analyzed from the layer projected DOS plots of silicene monolayer on these substrates. The LP-DOS plot of silicene monolayer on MT surface show that it can be metallic or magnetic behaviour, depending upon the choice of substrate. In FIG 2, we have shown the LP-DOS and band dispersion plots of some representative cases [viz. silicene on GaP(111), GaAs(111), ZnS(111) and ZnSe(111)], while for the same for $\operatorname{AlP}(111)$ and $\operatorname{AlAs}(111)$ are given as supplementary information [SI-FIG.1]. Silicene on MT surface of AlAs(111), AlP(111), GaAs(111) and ZnS(111) show metallic behavior with finite density of states at the Fermi level. FIG.2(b) and (c) show the LP-DOS and band dispersion plots of silicene on Ga terminated $\mathrm{GaAs}(111)$ and $\mathrm{Zn}$ terminated $\mathrm{ZnS}(111)$ substrate respectively. The LP-DOS of silicene on these substrates 
TABLE II. Magnetic Moment/ unit $\left(\mu_{B}\right)$ of the substrate $(4 \times 4)$, before and after introduction of Silicene monolayer on it. The subscript denotes the number of units in the substrate.

\begin{tabular}{|c|c|c|c|c|c|c|c|}
\hline NMT & $\begin{array}{l}\text { Mag. } \\
\text { Mom. }\end{array}$ & Si2@NMT & $\begin{array}{l}\text { Mag. } \\
\text { Mom. }\end{array}$ & MT & $\begin{array}{l}\text { Mag. } \\
\text { Mom. }\end{array}$ & Si2@MT & $\begin{array}{l}\text { Mag. } \\
\text { Mom. }\end{array}$ \\
\hline$(\mathrm{As} 6 \mathrm{Al6})_{4}$ & 1.41 & $(\mathrm{Si} 2 \mathrm{As} 6 \mathrm{Al6})_{4}$ & 1.45 & $(\mathrm{Al} 6 \mathrm{As} 6 \mathrm{H})_{4}$ & 0.84 & $(\mathrm{Si} 2 \mathrm{Al} 6 \mathrm{As} 6 \mathrm{H})_{4}$ & 0.00 \\
\hline$(\mathrm{P} 6 \mathrm{Al} 6)_{4}$ & 1.42 & $(\mathrm{Si2P} 6 \mathrm{Al} 6)_{4}$ & 1.48 & $(\mathrm{Al} 6 \mathrm{P} 6 \mathrm{H})_{4}$ & 0.80 & $(\mathrm{Si} 2 \mathrm{Al} 6 \mathrm{P} 6 \mathrm{H})_{4}$ & 0.00 \\
\hline$(\mathrm{As} 6 \mathrm{Ga} 6)_{4}$ & 0.75 & $(\mathrm{Si} 2 \mathrm{As} 6 \mathrm{Ga} 6)_{4}$ & 1.26 & $(\mathrm{Ga} 6 \mathrm{As} 6 \mathrm{H})_{4}$ & 0.65 & $(\mathrm{Si} 2 \mathrm{Ga} 6 \mathrm{As} 6 \mathrm{H})_{4}$ & 0.00 \\
\hline$(\mathrm{P} 6 \mathrm{Ga} 6)_{4}$ & 1.39 & $(\mathrm{Si} 2 \mathrm{P} 6 \mathrm{Ga} 6)_{4}$ & 1.49 & $(\mathrm{Ga} 6 \mathrm{P} 6 \mathrm{H})_{4}$ & 0.78 & $(\mathrm{Si} 2 \mathrm{Ga} 6 \mathrm{P} 6 \mathrm{H})_{4}$ & 0.86 \\
\hline$(\operatorname{Se} 6 \mathrm{Zn} 6)_{4}$ & 0.00 & $(\mathrm{Si} 2 \mathrm{Se} 6 \mathrm{Zn} 6)_{4}$ & 0.64 & $(\mathrm{Zn} 6 \mathrm{Se} 6 \mathrm{H})_{4}$ & 0.00 & $(\mathrm{Si} 2 \mathrm{Zn} 6 \mathrm{Se} 6 \mathrm{H})_{4}$ & 0.38 \\
\hline$(\mathrm{S} 6 \mathrm{Zn} 6)_{4}$ & 0.00 & $(\mathrm{Si} 2 \mathrm{~S} 6 \mathrm{Zn} 6)_{4}$ & 0.65 & $(\mathrm{Zn} 6 \mathrm{~S} 6 \mathrm{H})_{4}$ & 0.00 & $(\mathrm{Si} 2 \mathrm{Zn} 6 \mathrm{~S} 6 \mathrm{H})_{4}$ & 0.00 \\
\hline
\end{tabular}

resemble the overall DOS of free standing silicene monolayer with their Dirac cone shifted below the Fermi level by $0.7 \mathrm{eV}$ approximately. Silicene monolayer on MT surface of $\mathrm{GaP}(111)$, show magnetic behavior, with sharp spin up and spin down splitting near the Fermi level. The spin polarized LP-DOS and band dispersion plots of silicene on Ga terminated $\mathrm{GaP}(111)$ substrate is shown in FIG 2(a). It is to be noted here that in case of MT III-V substrates, the silicene bands comprises the valence band and conduction bands near the Fermi level. Therefore, the behaviour of the composite system is dictated by Silicene itself. The spin polarized LP-DOS plot of silicene monolayer on the MT surface of $\mathrm{ZnSe}(111)$ show half metallic behavior, with the spin down channel completely vanishing at the Fermi level FIG.2(d). However, in the band dispersion plot of the silicene-substrate composite system for substrates involving Zn [ie. ZnSe(111) and $\mathrm{ZnS}(111)]$, it is the $\mathrm{Zn} \alpha$-band predominantly constitutes the conduction band bottom and it protrudes below

TABLE III. Bader Charge Analysis of Silicon atoms of the silicene overlayer on MT and NMT substrates. +ve denotes electron lost while -ve denotes electron gained.

\begin{tabular}{lll|lll}
\hline MT & \multicolumn{5}{|l}{ NMT } \\
\hline System & Atom & Charge & System & Atom & Charge \\
\hline Si2Al6As6H & Si1 & -0.0475 & Si2As6Al6 & Si1 & +0.001 \\
& Si2 & -0.17 & & Si2 & +0.33 \\
Si2Al6P6H & Si1 & -0.05 & Si2P6Al6 & Si1 & +0.0375 \\
& Si2 & -0.16 & & Si2 & +0.225 \\
Si2Ga6As6H & Si1 & -0.005 & Si2As6Ga6 & Si1 & +0.015 \\
& Si2 & -0.255 & & Si2 & +0.2475 \\
Si2Ga6P6H & Si1 & +0.01 & Si2P6Ga6 & Si1 & +0.055 \\
& Si2 & +0.225 & & Si2 & +0.2125 \\
Si2Zn6Se6H & Si1 & +0.005 & Si2Se6Zn6 & Si1 & +0.0275 \\
& Si2 & -0.015 & & Si2 & +0.0575 \\
Si2Zn6S6H & Si1 & +0.2175 & Si2S6Zn6 & Si1 & +0.425 \\
& Si2 & -0.24 & & Si2 & -0.2375 \\
\hline
\end{tabular}

the Fermi level at $\Gamma$ point.

Results of the Bader charge analysis of Silicene monolayer on the MT semiconductor substrates is shown in TABLE-III In case of most of the MT substrates, electrons are transferred from the substrate to the silicene overlayer because of higher electronegativity of the $\mathrm{Si}$ atoms as compared to the contact metal atoms of the substrate. As a result of this the Silicene overlayer undergoes a substrate induced n-type doping [24, 25]. While only in case of $\mathrm{MT} \mathrm{GaP}(111)$ substrate, electrons are transferred from silicene to the substrate [ie. in the opposite direction] which suggest a p-type doping in the silicene overlayer similar to NMT cases [26, 27].

For a better understanding of the p-type/ n-type doping of the silicene overlayer, we have estimated the work function (WF) of MT/NMT $\left(\phi_{s}\right)$ surfaces of semiconductor substrates and compared it with that of free standing silicene monolayer $\left(\phi_{f l}\right)$. For $\phi_{s}>\phi_{f l}$, electrons are expected to transfer from silicene overlayer to the substrate, thereby leading to a p-type doping of the silicene overlayer. On the contrary, if $\phi_{f l}>\phi_{s}$, then electrons should be transferred from the substrate to the silicene overlayer leading to n-type doping. The results of our calculations shown in FIG 3, reveals that for MT substrates the WFs are consistently lower than that of free standing silicene (4.5 eV) with the exception of MT GaP(111) substrate (which is reverse to the other MT cases as also seen from the Bader analysis of TABLE-III). However, for NMT surfaces the trend is just reverse leading to the p-type doping. This is just reverse leading to the p-type doping. From the layer projected DOS plot of FIG,2, the difference between the substrate induced p-type and n-type doping of the silicene overlayer can be observed. In case of $\mathrm{GaP}(111)$, the p-type doping of the silicene overlayer is accompanied by shift in Fermi level towards the valence band (FIG 2a) while in the case of n-type doping (FIG 2p, c and d) the Fermi level shifts towards the conduction band. This type of p-type or n-type doping in silicene-substrate composite system could be useful for band gap engineering of bilayer silicene following the case of bilayer graphene [28, 29]. 


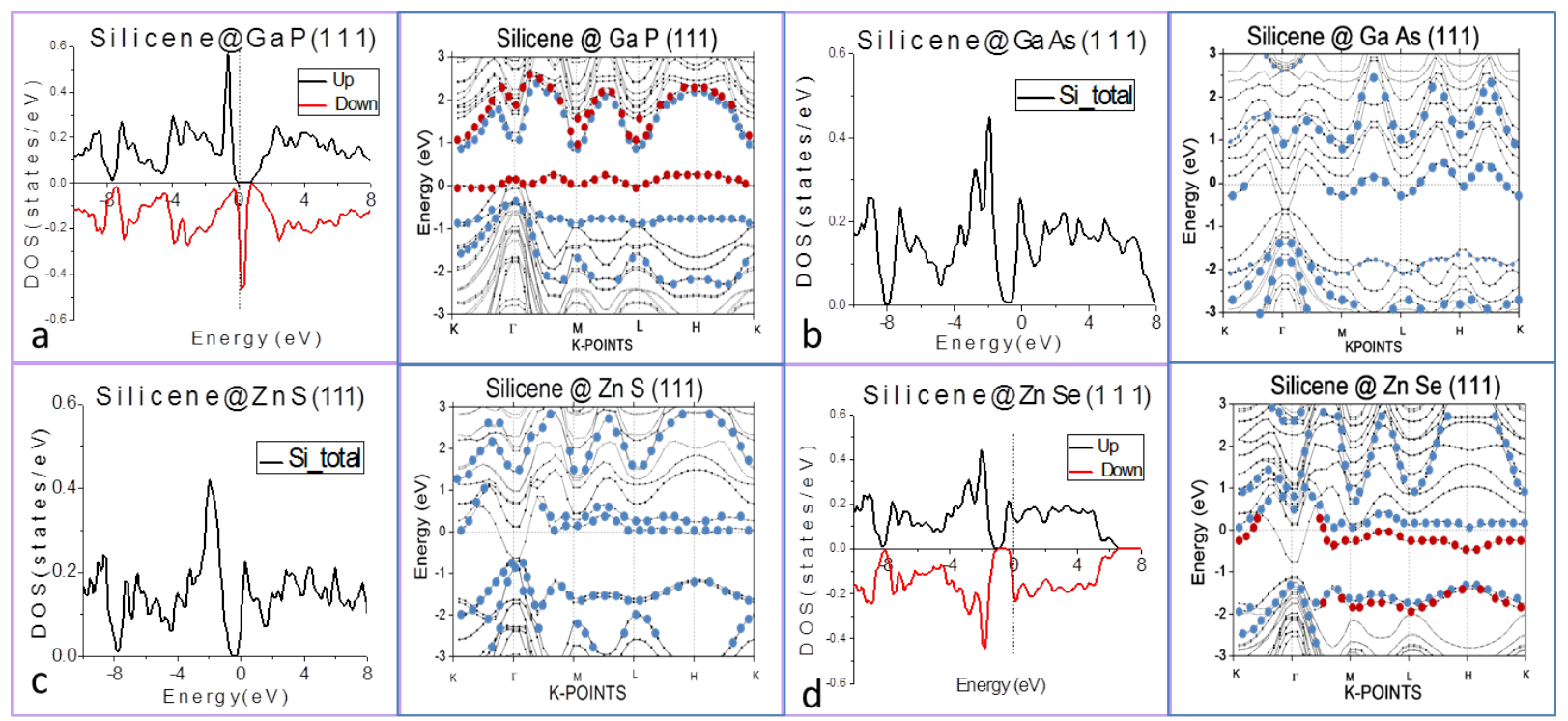

FIG. 2. Layer projected DOS plot and electronic band dispersion of Silicence on; (a): Ga terminated surface of GaP(111). The blue and red dotted bands corresponds to the spin up and spin down components of Si respectively. (b): Ga terminated surface of $\operatorname{GaAs}(111)$ substrate. The blue dotted bands corresponds to those of Silicene. (c): Zn terminated surface of ZnS(111) substrate. (d): Zn terminated surface of ZnSe(111) substrate. The spin down band (blue dotted) corresponds to the conduction band only while the spin up band (red dotted) has contribution in both the valence band and conduction band at the Fermi level giving rise to half metallic behaviour.

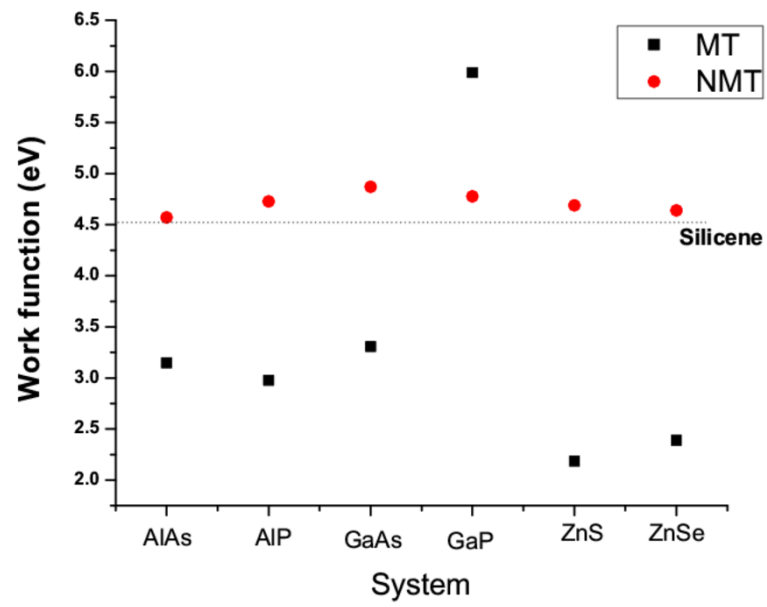

FIG. 3. Comparison of work function of $\mathrm{MT}(111)$ and $\operatorname{NMT}(111)$ semiconductor substrates. The dotted line corresponds to the work function of freestanding silicene monolayer

In conclusion, we report from our first principles based calculations, the bonding, stability and electronic structure of silicene monolayer, when epitaxially grown on various Group II-VI, Group III-V and Group IV semiconductor substrates viz. $\operatorname{AlAs}(111), \operatorname{AlP}(111), \operatorname{GaAs}(111)$, GaP(111), ZnS(111) and ZnSe(111). We find that the relative stability and other properties of the silicene overlayer depends sensitively on whether the interacting top layer of the substrate is metal or non-metal terminated. The binding energy of silicene monolayer to the metal terminated surfaces of these substrates are estimated to be range in the $0.56 \pm 0.12 \mathrm{eV} /$ atom. The introduction of silicene monolayer on the NMT surface of all these semiconductor substrates, leads to enhancement in the magnetic moment of the composite system. However, the behavior of silicene on MT surface of semiconductor substrates can be metallic, magnetic or semi-metallic depending on the choice of substrate. It undergoes substrate induced p-type doping on NMT substrates while n-type doping on MT substrates [with an exception of $\mathrm{GaP}(111)]$ depending upon its charge transfer with the substrates.

$\dagger$ presently affiliated to Fritz-Haber-Institut der Max Planck Gesellschaft, Faradayweg 4-6, Berlin-14195, Germany.

${ }^{*}$ Corresponding Author Email id: msgpd@iacs.res.in

[1] K. Takeda and K. Shiraishi, Phys. Rev. B 50, 14916 (1994).

[2] S. B. Fagan, R. J. Baierle, R. Mota, Z. J. R. da Silva and A. Fazzio, Phys. Rev. B 61, 9994 (2000).

[3] G. G. Guzmán-Verri and L. C. Lew Yan Voon, Phys. Rev. B 76, 075131 (2007). 
[4] A. K. Geim and K. S. Novoselov, Nat. Mater. 6, 183 (2007); K. S. Novoselov, A. K. Geim, S. V. Morozov, D. Jiang, Y. Zhang, S. V. Dubonos, I. V. Grigorieva, and A. A. Firsov, Science 306, 666 (2004).

[5] D. C. Elias, R. R. Nair, T. M. G. Mohiuddin, S. V. Morozov, P. Blake, M. P. Halsall, A. C. Ferrari, D. W. Boukhvalov, M. I. Katsnelson, A. K. Geim and K. S. Novoselov, Science 323, 610 (2009).

[6] K. S. Novoselov, A. K. Geim, S. V. Morozov, D. Jiang, M. I. Katsnelson, I. V. Grigorieva, S. V. Dbonos and A. A. Firsov, Nature (London) 438, 197 (2005).

[7] B. Aufray, A. Kara, S. Vizzini, H. Oughaddou, C. Léandri, B. Ealet, G. Le Lay, Appl. Phys. Lett. 96183102 (2010).

[8] B. Lalmi, H. Oughaddou, H. Enriquez, A. Kara, S. Vizzini, B. Ealet and B. Aufray, Appl. Phys. Lett 97,223109 (2010).

[9] P. De Padova, C. Quaresima, C. Ottaviani, P.M. Sheverdyaeva, P. Moras, C. Carbone, D. Topwal, B. Olivieri, A. Kara, H. Oughaddou, B. Aufray, G. Le Lay, Appl. Phys. Lett. 96261905 (2010).

[10] A. Kara, C. Léandri, M.E. Dévila, P. De Padova, B. Ealet, H. Oughaddou, B. Aufray and G. Le Lay, J. Supercond. Novel Magn. 22259 (2009).

[11] P. Vogt, P. D. Padova, C. Quaresima, J. Avila, E. Frantzeskakis, M. C. Asensio, A. Resta, B. Ealet and G. L. Lay, Phys. Rev. Lett. 108, 155501 (2012).

[12] B. Feng, Z. Ding, S. Meng, Yugui Yao, X. He, P. Cheng, L. Chen and K. Wu, Nano Lett., 12, 3507 (2012).

[13] A. Kara, S. Vizzini, C. Leandri, B. Ealet, H. Oughaddou, B. Aufray and G. Le Lay, J. Phys.: Condens. Matter, 22, 045004 (2010).

[14] A. Kara, H. Enriquez, Ari P. Seitsonen, L.C. Lew Yan Voone, S. Vizzini , B. Aufray , H. Oughaddoub, Surface
Science Reports 67, 1 (2012).

[15] A. Fleurence, R. Friedlein, T. Ozaki, H. Kawai, Y. Wang, and Y. Yamada-Takamura, Phys. Rev. Lett. 108, 245501 (2012).

[16] L. Meng, Y. Wang, L. Zhang, S. Du, R. Wu, L. Li, Y. Zhang, G. Li, H. Zhou, W. A. Hofer and H. Gao, Nano Lett. 13, 685 (2013).

[17] L. Chen, H. Li, B. Feng, Z. Ding, J. Qiu, P. Cheng, K. Wu and S. Meng, Phys. Rev. Lett. 110, 085504 (2013).

[18] P. Hohenberg and W. Kohn, Phys. Rev. B 136, 864 (1964).

[19] W. Kohn and L. Sham, Phys. Rev. A 140, 1133 (1965).

[20] G. Kresse and J. Hafner, Phys. Rev. B 49, 14251 (1994); G. Kresse and J. J. Fürthmller, Comput. Mater. Sci. 6, 15 (1996).

[21] P. E. Blöchl, Phys. Rev. B 50, 17953 (1994).

[22] J. P. Perdew, K. Burke and M. Ernzerhof, Phys. Rev. Lett. 77, 3865 (1996).

[23] P. Pulay, Chem. Phys. Lett. 73, 393 (1980).

[24] W. Chen, S. Chen, D. C. Qi, X. Y. Gao and A. T. S. Wee, J. Am. Chem. Soc., 129, 10418 (2007).

[25] N. Jung, N. Kim, S. Jockusch, N. J. Turro, P. Kim and L. Brus, Nano Lett., 9, 4133 (2009).

[26] J. H. Chen, C. Jang, S. Adam, M. S. Fuhrer, E. D. Williams and M. Ishigami, Nat. Phys., 4, 377 (2008).

[27] A. Bostwick, T. Ohta, T. Seyller, K. Horn and E. Rotenberg, Nat. Phys. 3, 36 (2007).

[28] Y. Zhang, T. Tang, C. Girit, Z. Hao, M. C. Martin, A. Zettl, F. Michael, C. Y. R. Shen and F. Wang, Nature 459, 820 (2009).

[29] T. Ohta, A. Bostwick, T. Seyller, K. Horn and E. Rotenberg, Science 313, 951 (2006). 